\title{
Magnitude and factors associated with appropriate complementary feeding among mothers having children 6-23 months-of-age in Northern Ethiopia; a community-based cross-sectional study
}

\author{
Ergib Mekbib ${ }^{1}$, Ashenafi Shumey ${ }^{2,}$, Semaw Ferede $^{2}$, Fisaha Haile ${ }^{2}$ \\ ${ }^{1}$ Health insurance agency Mekelle branch, Mekelle, Ethiopia \\ ${ }^{2}$ Public Health Department, College of Health Sciences, Mekelle University, Mekelle, Ethiopia \\ Email address: \\ ashureech@yahoo.com (A. Shumey)
}

To cite this article:

Ergib Mekbib, Ashenafi Shumey, Semaw Ferede, Fisaha Haile. Magnitude and Factors Associated with Appropriate Complementary Feeding among Mothers Having Children 6-23 Months-of-Age in Northern Ethiopia; A Community-Based Cross-Sectional Study. Journal of Food and Nutrition Sciences. Vol. 2, No. 2, 2014, pp. 36-42. doi: 10.11648/j.jfns.20140202.13

\begin{abstract}
Background- Adequate and appropriate complementary feeding during 6-23 months-of-age, is fundamental to the development of each child's full human potential. Ethiopian national survey reports showed the minimum acceptable diet was $4.2 \%$ in same region. But in most cases only timely initiation is considered as the only indicator of complementary feeding. The aim of this study was to assess the prevalence of appropriate complementary feeding practices and associated factors among mothers having 6 - 23 months of age children in Northern Ethiopia. Methods- A community-based crosssectional study design was conducted among 428 mothers who had children with 6-23 months of age in Northern Ethiopia. Simple random sampling was used to select the required number of sample. Pretest was done among 22 respondents out of the study area. A face-to-face interview was used to collect data using structured questionnaire. Data were entered with EPI info version 3.5.1 and cleaning and analysis was done by using SPSS version 16. Frequencies distribution, binary and multiple logistic regressions were done. OR with $95 \%$ confidence interval was computed to measure the strength of association. Results - The response rate was $98.6 \%$. In this study only $10.75 \%(95 \% \mathrm{CI}=8.07,13.95)$ children aged $6-23$ months received appropriate complementary feeding. Child's age ( $\mathrm{AOR}=4.21)$, education level of mother $(\mathrm{AOR}=3.84)$, and postnatal care follow up ( $\mathrm{AOR}=2.80$ ) were found to be independent predictor of timely initiation of complementary feeding. Conclusion and recommendations - one out of ten mothers fed complementary foods appropriately to their children aged 623 months which was very low. Mothers who are illiterate and completed only primary school need more attention. All mothers must be encouraged to make postnatal care follow up.
\end{abstract}

Keywords: Complementary Feeding, Food Diversity, Meal Frequency, Minimum Acceptable Diet

\section{Background}

Adequate nutrition during infancy and early childhood is fundamental to the development of each child's full human potential. World Health Organization recommends introducing complementary foods when an infant reaches 6 months of age. After sixth month of age, breast milk alone is no longer sufficient to meet the nutritional requirements of 623 months of age children [1-6].

Inappropriate complementary feeding practices remain as major public health problem in many developing countries where many children are victim of the malpractice [7]. Less than one-third of 6-23 months old children met the minimum criteria for dietary diversity, and only $50 \%$ received the minimum number of meals. When these indicators combined, called as the minimum acceptable diet, only $21 \%$ of children aged 6-23 months met the minimum criteria [7]. According to Ethiopian demographic and health survey and alive thrive Ethiopia survey in 2011, in Tigray regional state the percentage of minimum acceptable diet was $4.2 \%$. This indicates that appropriate complementary feeding practice in this area was very low $[8,9]$.

Inappropriate complementary feeding practices continue to make children to be vulnerable to irreversible outcomes of stunting, poor cognitive development and significantly 
increased risk of infectious diseases leading to gastroenteritis, diarrhea and acute respiratory infection [10-13]. Globally, malnutrition has been responsible, directly or indirectly, for $60.0 \%$ of the 10.9 million deaths annually among children under five. Well over two-thirds of these deaths, which are often associated with inappropriate feeding practices, occur during the first year of life [5]. It is believed that appropriate $\mathrm{CF}$ has the potential to prevent $6 \%$ of all under-five deaths particularly in the developing world $[14,15]$.

The study was purposely conducted in area which was considered to have higher magnitude of timely initiation of complementary feeding to enable us to see the gap that could exist between appropriate complementary feeding, considering other criteria, and timely initiation. The aim of this study was therefore: i) to determine the prevalence of timely initiation of complementary feeding practices among mothers having children 6-23 months of age in Northern Ethiopia; ii)to determine the prevalence of appropriate complementary feeding practices among mothers having children 6-23 months of age in Northern Ethiopia; iii) to identify factors affecting complementary feeding practices among mothers having children 6-23 months of age in Northern Ethiopia.

\section{Methods}

\subsection{Study Area and Period}

The study was carried out in Abyi-Adi town, which is located $95 \mathrm{kms}$ Northwest of Mekelle, the capital of Tigray regional state and $978 \mathrm{Kms}$ North of Addis Ababa, the capital of Ethiopia. The total population is estimated to be 18,319. Among this population, 9343(51\%) were females, $8976(49 \%)$ male, $2725(15 \%)$ under 5, 1496(8\%) under 3 and $663(3.6 \%)$ children aged 6-23 months. The dominant religion is orthodox Christian followed by Muslim. There is one governmental hospital and one health center in the town. The study was conducted from October 2012 to May 2013. It is a town which presumably is believed to have good practice of complementary feeding considering timely initiation as the only indicator.

\subsection{Study Design, Population, Sampling}

A community-based cross-sectional was conducted. Mothers of infants aged from 6-23 months in Abyi-Adi were the study population. The sample size for the study was calculated using Epi Info statCalc for unmatched case control formula. The following assumption were used; Power $=80 \%, Z \beta=.84$, significance level $=5 \%, Z \alpha=1.96$, to detect an odds ratio of 2.0 between the two groups and $69.2 \%$ mothers exposed to media [29] was take as population exposed to a factor, ratio of controls to cases 1 (equal number of cases and controls. Considering $10 \%$ none response rate, a total of 434 mothers were required for the study. Total number of children in the age group between 623 months in the town was taken from the Health centre and Hospital EPI registration books, where EPI coverage was
$100 \%$. Then sampling frame was prepared. Finally, the required number of mothers were selected using simple random sampling.

\subsection{Data Collection Procedure and Tools}

Data were collected using face-to-face interview during house-to-house visit from mothers who had 6-23 months age children using structured questionnaire. The questionnaire were originally prepared in English the translated to local language. The questionnaire comprises three parts; the first part is about background characteristics of mothers and children, the second includes maternal health related practices and the third includes feeding practices of the child. To proceed with data collection, six diploma nurses as data collector and one BSC nurse as supervisor were recruited.

Data quality control: The questionnaire was translated to local language, Tigrigna, and then back to English by two people for consistency. Pretest was made in $22(5 \%)$ mothers in the study area, which was not included in the study to assess the content and approach of the questionnaire. Training was given to data collectors and supervisor for two days on the objective, relevance of the study, confidentiality of information, respondent's right, about pre-test, informed consent and techniques of interview; after the investigators discuss deeply. All questioners were checked by the supervisor daily for completeness and the principal investigator had monitored the overall quality of data collection. Besides this, the investigators carefully entered and thoroughly cleaned the data before the commencement of the analysis.

\subsection{Standard and Operational Definitions}

Timely introduction of complementary feeding: proportion of children 6-23 months of age who started complementary foods at 6 th month.

Minimum dietary diversity: proportion of children 6-23 months of age who receive foods from four or more food groups during the previous day. The seven food groups used for tabulation of this indicator were: grains, roots and tubers; legumes and nuts; dairy products (milk, yogurt, cheese); flesh foods (meat, chicken and liver/organ meats); eggs; vitamin Arich fruits and vegetables; and other fruits and vegetables.

Minimum meal frequency: proportion of breastfed and non-breastfed children 6-23 months of age who receive solid, semi-solid or soft foods the minimum number of times or more (minimum is defined as: two times for breastfed infants 6-8 months; three times for breastfed children 9-23 months; and four times for non-breastfed children 6-23 months) in the previous day.

Minimum acceptable diet: proportion of breastfed children 6-23 months of age who had at least the minimum dietary diversity and the minimum meal frequency during the previous day, and non-breastfed children 6-23 months of age who received at least two milk feedings and had at least the minimum dietary diversity not including milk feeds and the minimum meal frequency during the previous day. 


\subsection{Complementary Feeding Practices}

- $\quad$ Appropriate- If the mother responds correctly all the above four indicators, as recommended.

- Inappropriate - Among the four indicators if at least one indicator was not fulfilled.

\subsection{Data Analysis, Presentation and Interpretation}

Data were entered using EPI info version 3.5.1 statistical software and cleaning and analysis was made using SPSS version 16. Cleaning was made using frequencies. Univariate analysis was done to describe dependent and independent variables; percentages, frequency distributions and measures of central tendency and measures of dispersion were used for describing data. Then binary logistic regression was made to see the crude significant relation of each variable with dependant variables. Finally, independent variables found significant were entered to multivariate logistic regressions to control the effect of confounding. Stepwise backward LR was used for multiple logistic regressions. Odds ratio with $95 \%$ confidence interval to ascertain association between independent and dependent variable was used.

\subsection{Ethical Considerations}

Ethical clearance was obtained from institutional review board of Mekelle University. The offices of Tigray Region Health Bureau, Mekelle zonal health Department and the selected Health institutions were communicated with formal letters from the Mekelle University, Department of Public Health.

All the study participants were reassured that they would be anonymous. Names or any personal identifiers were not recorded. Respondents were clearly told about the study and the variety of information needed from them. They were given the chance to ask anything about the study and made free to refuse or stop the interview at any moment they want if that was their choice. After interview the importance of timely initiation of complimentary feeding was discussed with the respondents who had not had knowledge on complementary issues.

\section{Results}

\subsection{Socio-Demographic Characteristics of Mothers}

Of the 434 sampled mothers, 428 were successfully included in the study making the response rate of $98.6 \%$. Biological mothers accounted for 422 (98.6\%) of caregivers, while $6(1.4 \%)$ were other caregivers such as grand-mothers and sisters. The median age of mothers was 26 years with inter quartile range (IQR) being 8 . Three hundred ninety one (91.4\%) were orthodox by religion and all of them (100\%) belong to the Tigray ethnic group (from the same region). Concerning the educational status of mothers, $310(72.4 \%)$ had attended formal school. The majority of mothers, 363 (84.4\%), were married and 264
(61.7\%) were house wives by occupation. More than a third, 161 (37.6\%), of mothers earned an average monthly income of less than or equal to 999 Ethiopian Birr $(<55$ USD). Husbands of $317(85.9 \%)$ mothers had attended formal education. The median age of children was 13 months with IQR being 8 (Table 1).

\subsection{Obstetrics and Health Service Related Variables}

Almost all, 424 (99.1\%), mothers had antenatal care follow up at least once during the last pregnancy. About $66 \%(279 / 428)$ of mother had at least 4 visits as recommended. About $86.0 \%$ of mothers gave birth their last child at health institution. Approximately $70.0 \%$ had received postnatal care (PNC) at least once (Table 1).

\subsection{Complementary Feeding Knowledge and Practices}

In this study, it was found that majority (99.1\%) of mothers had ever practiced breastfeeding. Approximately $87 \%(371 / 428)$ of mothers had satisfactory knowledge and the rest $13 \%$ had poor knowledge about complementary feeding. About 76\% (324/428) of mothers had supportive attitude while the rest $24 \%$ had no supportive attitude towards complementary feeding.

Grain, roots and tubers were the most commonly taken food items by the children in 24 hours preceding the survey. Legumes and nuts (28.9\%), vitamin-A rich foods (26.3\%), other fruits and vegetables (60.9\%), flesh foods (16.4\%) and egg (25.8\%) was higher in the 18-23 months group compared to the other groups. Generally, the rates of different food groups offered during the past 24 hours were uniformly lower in the 6-11 months age group, with the lowest rates reported for flesh foods (3.4\%) (Table 2).

Approximately $79.7 \%$ (95\% CI: $75.66 \%-83.28,341 / 428)$ of mothers introduced complementary feeding at 6 months age of the children as per recommended. Only $9(2.1 \%)$ mothers introduced complementary feeding early before 6 month, 68 $(15.9 \%)$ mothers initiated late after 6 month and $10(2.3 \%)$ mothers did not start complementary feeding at all.

Only seventy six (17.8\%) mothers offered four or more food groups to their child meeting the minimum dietary diversity criteria on the day preceding the study. One hundred seventy one $(40.0 \%)$ mothers fed their children more than two times the day preceding the study. Only 51(11.9\%) of mothers had practice the minimum acceptable diet.

The overall prevalence of appropriate complementary feeding practices, combining the four mentioned indicators was $10.75 \%(95 \% \mathrm{CI}=8.07,13.95,46 / 428)$.

\subsection{Factors Found Associated with Appropriate Complementary Feeding Practice}

After applying bivariate and multiple logistic regressions, three variables were found to be significantly associated with appropriate complementary feeding practice. These were child's age 18-23 months ( $\mathrm{AOR}=4.21$ ), mothers who had postnatal care $(\mathrm{AOR}=2.80)$ and secondary school or above educated mothers $(\mathrm{AOR}=3.84)$ were more likely to give 
appropriate complementary feeding to their children (Table 3).

Table 1. Socio-demographic and obstetrics and health related variables of mothers who had children 6-23 months of age ( $n=428)$ in Abyi-Adi town, Tigray, Northern Ethiopia, October 2012 to May 2013.

\begin{tabular}{|c|c|c|c|}
\hline Variable & Categories & Frequency & Percentage \\
\hline \multirow{5}{*}{ Mothers' age } & $<20$ & 35 & 8.2 \\
\hline & $20-24$ & 112 & 26.2 \\
\hline & $25-29$ & 146 & 34.1 \\
\hline & $30-34$ & 77 & 18.0 \\
\hline & $\geq 35$ & 58 & 13.6 \\
\hline \multirow{2}{*}{ Marital status } & Married & 363 & 84.8 \\
\hline & Divorced/Widowed/Separated & 65 & 15.2 \\
\hline \multirow{3}{*}{ Religion } & Orthodox & 391 & 91.4 \\
\hline & Muslim & 37 & 8.6 \\
\hline & No education & 118 & 27.6 \\
\hline \multirow[t]{2}{*}{ Mothers' educational status } & Primary education (1-8) & 126 & 29.4 \\
\hline & Secondary education (9+) & 184 & 43.0 \\
\hline \multirow{2}{*}{ Mothers' occupation } & Unemployed & 264 & 61.7 \\
\hline & Employed & 164 & 38.3 \\
\hline \multirow[t]{5}{*}{ Monthly income (ETB) } & $\leq 999$ & 161 & 37.6 \\
\hline & 1000-1999 & 143 & 33.4 \\
\hline & 2000-2999 & 65 & 15.2 \\
\hline & $3000-3999$ & 41 & 9.6 \\
\hline & $\geq 4000$ & 18 & 4.2 \\
\hline \multirow[t]{3}{*}{ Child's age (months) } & $6-11$ & 148 & 34.6 \\
\hline & $12-17$ & 152 & 35.5 \\
\hline & $18-23$ & 128 & 29.9 \\
\hline \multirow[t]{3}{*}{ Number of children } & 1 & 173 & 40.4 \\
\hline & $2-4$ & 233 & 54.4 \\
\hline & 5 and above & 22 & 5.1 \\
\hline \multirow[t]{2}{*}{ Number of ANC follow up } & $<4$ & 145 & 34.2 \\
\hline & $\geq 4$ & 279 & 65.8 \\
\hline \multirow[t]{2}{*}{ Place of delivery } & Home & 60 & 14.0 \\
\hline & Health institution & 368 & 86.0 \\
\hline \multirow[t]{2}{*}{ Postnatal care } & Yes & 299 & 69.9 \\
\hline & No & 129 & 30.1 \\
\hline
\end{tabular}

*private employee, student, farmer

Table 2. Types of food given to children aged 6-23 months by age group (n=428), Abyi-Adi town, Tigray, Northern Ethiopia, October 2012 to May 2013.

\begin{tabular}{|c|c|c|c|c|c|c|}
\hline \multirow{3}{*}{ Food groups } & \multicolumn{6}{|c|}{ Age of child in month } \\
\hline & \multicolumn{2}{|c|}{$6-11$} & \multicolumn{2}{|c|}{$12-17$} & \multicolumn{2}{|c|}{$18-23$} \\
\hline & Yes $(\%)$ & No (\%) & Yes $(\%)$ & No (\%) & Yes $(\%)$ & No $(\%)$ \\
\hline Grain,root and tubers & 86.5 & 13.5 & 95.4 & 4.6 & 96.1 & 3.9 \\
\hline Legumes and nuts & 22.3 & 77.7 & 27.0 & 73.0 & 28.9 & 71.1 \\
\hline Dairy products & 16.9 & 83.1 & 27.6 & 72.4 & 23.4 & 76.6 \\
\hline Vit A rich foods & 8.8 & 91.2 & 16.4 & 83.6 & 26.3 & 79.7 \\
\hline Other fruits and vegtables & 41.2 & 58.8 & 52.0 & 48.0 & 60.9 & 39.1 \\
\hline Flesh foods & 3.4 & 96.6 & 8.6 & 91.4 & 16.4 & 83.6 \\
\hline Egg & 18.2 & 81.8 & 24.3 & 75.7 & 25.8 & 74.2 \\
\hline
\end{tabular}


Table 3. Factors associated with appropriate complementary feeding practice among mothers who had 6-23 months children in Abyi-Adi town, Tigray, Northern Ethiopia, October 2012 to May 2013.

\begin{tabular}{|c|c|c|c|c|}
\hline \multirow{2}{*}{ Variables } & \multicolumn{2}{|c|}{ Complementary feeding practice } & \multirow{2}{*}{ COR $[95 \%$ C.I] } & \multirow{2}{*}{ AOR [95\% C.I] } \\
\hline & Appropriate N (\%) & Inappropriate N (\%) & & \\
\hline Mothers' education & $5(4.2)$ & 113(95.8) & 1.00 & 1.00 \\
\hline No education & $10(7.9)$ & $116(92.1)$ & $1.94[0.64,5.87]$ & $1.81[0.57,5.70]$ \\
\hline Primary education & $31(16.8)$ & $153(83.2)$ & $4.57[1.72,12.14]^{* *}$ & $3.84[1.33,11.09]^{*}$ \\
\hline \multirow{2}{*}{\multicolumn{4}{|c|}{ Mothers' occupation }} & \\
\hline & $22(8.3)$ & 242(91.7) & 1.00 & 1.00 \\
\hline Unemployed & $24(14.6)$ & $140(85.4)$ & $1.88[1.02,3.48]^{*}$ & $1.38[0.67,2.83]$ \\
\hline \multicolumn{5}{|l|}{ Age of child (months) } \\
\hline $6-11$ & $8(5.4)$ & 140(94.6) & 1.00 & 1.00 \\
\hline $12-17$ & $15(9.9)$ & 137(90.1) & $1.91[0.78,4.66]$ & $1.94[0.76,4.94]$ \\
\hline $18-23$ & $23(18.0)$ & $105(82.0)$ & $3.83[1.64,8.90]^{* *}$ & $4.21[1.70,10.39]^{* *}$ \\
\hline \multicolumn{5}{|l|}{ Monthly income (ETB) } \\
\hline$\leq 999$ & $15(9.3)$ & 146(90.7) & 1.00 & 1.00 \\
\hline $1000-1999$ & $7(4.9)$ & 136(95.1) & $0.50[0.19,1.26]$ & $0.45[0.16,1.20]$ \\
\hline $2000-2999$ & $9(13.8)$ & $56(86.2)$ & $1.56[0.64,3.77]$ & $1.04[0.40,2.72]$ \\
\hline $3000-3999$ & $10(24.4)$ & $31(75.6)$ & $3.14[1.29,7.63]^{*}$ & $2.26[0.86,5.98]$ \\
\hline$\geq 4000$ & $5(27.8)$ & $13(72.2)$ & $3.74[1.17,11.94]^{*}$ & $2.54[0.70,9.19]$ \\
\hline \multicolumn{5}{|l|}{ Owns television } \\
\hline No & $8(5.4)$ & $141(94.6)$ & 1.00 & $\begin{array}{c}1.00 \\
0.025017\end{array}$ \\
\hline Yes & $38(13.6)$ & $241(86.4)$ & $2.79[1.26,6.12]^{*}$ & $2.09[0.84,5.21]$ \\
\hline No. ANC visit & $15(10.3)$ & $130(89.7)$ & 1.00 & \\
\hline $\begin{array}{l}<4 \\
>4\end{array}$ & $31(11.1)$ & $248(88.9)$ & $1.08[0.56,2.07]$ & \\
\hline \multicolumn{5}{|l|}{ Place of delivery } \\
\hline Home & $3(5.4)$ & $53(94.6)$ & 1.00 & \\
\hline Health care facility & $43(11.6)$ & $329(88.4)$ & $2.30[0.69,7.71]$ & \\
\hline PNC follow up & & & & \\
\hline No & $40(134)$ & $259(866)$ & $3.16[130766]^{1.00}$ & $280[1.00$ \\
\hline Yes & $40(13.4)$ & $259(86.0)$ & $3.10[1.30,1.06]^{*}$ & $2.80[1.11,1.03]^{*}$ \\
\hline \multicolumn{5}{|c|}{ Attitude towards complementary } \\
\hline feeding & $5(4.8)$ & $99(95.2)$ & 1.00 & 1.00 \\
\hline unsupportive attitude & $41(12.7)$ & 283(87.3) & $2.86[1.10,7.46]^{*}$ & $1.74[0.62,4.88]$ \\
\hline supportive attitude & & & & \\
\hline
\end{tabular}

$* \mathrm{P}<0.05, * * \mathrm{P}<0.01, \mathrm{COR}$ (Crude odds ratio), AOR (Adjusted odds ratio), CI (Confidence interval)

\section{Discussion}

The result of our study revealed that the prevalence of appropriate complementary feeding was $10.75 \%$. Educated mothers, children age 18-23 months and mothers who had postnatal care were factors that can increase appropriate complementary feeding practice.

About $80 \%$ of mothers had started complementary feeding at sixth month of the child's age, as recommended. It was higher than the findings from United Arab Emirates $(<17 \%)$, Delhi India (17.5\%), Mekelle (62.8\%), Ethiopia with national level (51\%) and Harar (54.4\%) [16-20]. This relatively higher prevalence might be due to practices change with time, better postnatal care follow up and the effort of health extension worker in the area.

Minimum acceptable diet, a combination of minimum meal frequency and minimum dietary diversity, in this study was $11.9 \%$, which was higher than in Ethiopian national level result (5.2\%) and Tigray (4.2\%) [8] but was lower than the findings from Sirlanka (68\%), Bangladesh (40\%), Nepal (32\%), Zambia (25.1\%), and Tanzania $(15.9 \%)$ [21-25]. This low level of frequency might be attributed by low maternal literacy, different culture and lower economy.

Only $10.75 \%$ of mothers were practicing appropriate complementary feeding which was lower than other studies in Sirlanka, Bangladesh, Nepal, Zambia, and Tanzania where it was above 20 [21-25]. This might be due to poor socioeconomic status, lower maternal literacy and shortage of nutrition professional. Health extension workers were usually concerned on timely initiation of complementary feeding than dietary diversity and frequency of meal.

There was a huge difference between the timely imitation alone and appropriate complementary feeding considering other criteria. However, in most of the cases the time of initiation of complementary feeding is reported as the only important indicator of complementary feeding, though the fact showed the presence of quite significant difference.

There was also a big gap between the knowledge of mothers about appropriate complementary feeding $(86.7 \%)$ and their practices $(10.75 \%)$. This might indicate the presence of some cultural practice that could affect complementary feeding and poor socioeconomic status or pressure by other part of the family on the mothers. 
Children within the age group 18-23 months were 4.2 times more likely to be appropriately fed as compared to infants in the age group 6-11 months. This result was in congruent with the findings in Nepal, Indonesia and Tanzania [24-,26]. This might be due to the misunderstanding of mothers that young children could not be able to digest food like meat and egg. In addition to this some of the mothers couldn't introduce complementary feeding at six month.

Mothers who had educational status of secondary and above were 3.8 times more likely to practice appropriate complementary feeding than those mothers who had no education. This result was consistent with the findings Bangladesh, India, Indonesia, Sirlanka, Nepal, Tanzania and Nairobi Kenya [21-27] where mothers with little or no education were a significant predictor of inappropriate complementary feeding practice. This might be because of educated mothers would have knowledge about the importance of appropriate complementary feeding practice, better understanding of information. Furthermore, education might enhance the status of mother and enable them to develop greater confidence and capacity to make decisions about their child feeding practice. At the same time, educated mother may be influenced by media advertising of complementary feeding.

Mothers who followed postnatal care service were 2.8 times more likely to practice appropriate complementary feeding than those who did not follow the service. This result is similar with the findings in Sirlinka, India and Tanzania $[17,21,25]$ where lack of post-natal contacts was a significant predictor of inappropriate complementary feeding practice. This might be due to the result of information and counseling that the mothers received from health workers during their postnatal visits.

On the other hand, this study revealed that there was no association between number of antenatal care visit and appropriate complementary feeding practice and it disagrees with the study in Sirlinka, Nepal, India and Harar $[17,19,21,23]$ where inadequate antenatal care was associated with inappropriate complementary feeding. This could show health professionals providing antenatal care were more focused on the pregnancy related information than child health care practices.

In this study there was no association between television ownership and appropriate complementary feeding practice. This result disagreed with the study in Nepal and India $[17,23]$. This was because nutrition related information was rarely broadcasted via Ethiopian television. .

\section{Conclusion}

One out of ten mothers fed complementary foods appropriately to their children aged 6-23 months which was very low. About $90 \%$ mothers were not feeding complementary foods appropriately considering, timely introduction, minimum dietary diversity, minimum meal frequency and minimum acceptable diet, which would have negative implication on the health of infants and young children. This study indicated the importance of immediate action to promote appropriate complementary feeding. Educated mothers, children age 18-23 months and mothers who had postnatal care were factors that can increase appropriate complementary feeding practice.

\section{We Recommend}

It is important to evaluate the effectiveness, approaches and coverage of nutrition interventions being implemented to promote complementary feeding practice.

$>$ Expanding education opportunities for women and incorporating related information in primary school is worthy.

> Infant and young child feeding practice should not be measured by considering only timely introduction of complementary feeding but also minimum dietary diversity, minimum meal frequency and minimum acceptable diet.

> Maternal health service outlets should be used to transmit child feeding information and hence, training should be given for all health workers to focus on minimum dietary diversity and minimum meal frequency as well.

> Strengthening inter-sectoral collaboration may be urgent and needed to think of the possibilities to increase and supply the minimum variety of food staples.

\section{Competing Interests}

The authors declare that they have no competing interests.

\section{Acknowledgements}

We are thankful to all respondents for their willingness to participate in the study, as it is through their cooperation that we have been able to write this report.

The data collectors are acknowledged for their effort in collecting the information. We also would like to thank to Mekelle University for the financial support and technical assistance and approving this study.

\section{References}

[1] Federal Ministry of Health, Family Health Department Ethiopia: National strategy for infant and young child feeding. Addis Ababa: Federal Ministry of Health, Family Health Department Ethiopia; 2004.

[2] Martines J, Paul VK, Bhutta ZA, Koblinsky M, Soucat A, Walker N, et al: Neonatal survival: a call for action. Lancet 2005, 365:1189-1197.

[3] Singer PA, Ansett S, Sagoe-Moses I: What could infant and young child nutrition learn from sweatshops? BMC Public Health 2011, 11:276. 
[4] PAHO/WHO: Guiding Principles for Complementary Feeding of the Breastfed Child. Division of Health Promotion and Protection. Food and Nutrition Program. Washington/Geneva: Pan American Health Organization/World Health Organization; 2003.

[5] World Health Organization: Global strategy for infant and young child feeding. Geneva: World Health Organization; 2003:41.

[6] Imdad A, Yakoob MY, Bhutta ZA: Impact of maternal education about complementary feeding and provision of complementary foods on child growth in developing countries. BMC Public Health 2011, 11(Suppl 3):S25.

[7] Chessa K. Lutter, Bernadette M. E. G. Daelmans, Mercedes de Onis, Undernutrition, Poor Feeding Practices, and Low Coverage of Key Nutrition Interventions. Pediatrics 2011; 128 ; e1418. http://pediatrics.aappblications.org/content/128/6/e1418.full. html.

[8] Ali D, Tedla M, Subandoro A, Bamezai A, Rawat R, Menon P. Alive \& Thrive Baseline Survey Report: Ethiopia. Washington, D.C.: Alive \& Thrive, 2011.

[9] Central Statistical Agency [Ethiopia] and ORC Macro: Ethiopia Demographic and Health Survey 2011. Addis Ababa, Ethiopia and Calverton, Maryland, USA: Central Statistical Agency and ORC Macro; 2012.

[10] WHO and UNICEF: Complementary feeding of young children in developing countries: a review of current scientific knowledge. Geneva: World Health Organization, WHO/NUT/98; 1998:1.

[11] Saha KK, Frongillo EA, Alam DS, Ariffen SE, Persson LA, Rasmussen KM: Appropriate infant feeding practices result in better growth of infants and young children in rural Bangladesh. Am J Clin Nutr 2008, 87:1852-1859.

[12] World Health Organization, Division of Family Health: The prevalence and duration of breastfeeding in urban population of Chandigarh during adecade. Indian Pediator 1987, 24:879-887.

[13] Hop LT, Gross R, Giay T, Sastroamidjojo S, Schultink W, Lang NT: Premature complementary feeding is associated with poorer growth of Vietnamese children. J Nutr 2000, 130:2683-2690.

[14] Gareth J, Richard W, Robert E, et al. How many child deaths can we prevent this year? The lancet 2003; 362:65-67.

[15] Lutter C. Meeting the challenges to improve complementary feeding. Standard Committee on Nutrition News. 2003; 27:4-9

[16] Radwan: Patterns and determinants of breastfeeding and complementary feeding practices of Emirati Mothers in the United Arab Emirates. BMC Public Health 2013 13:171.
[17] Aggrawal A, Verma S, Feridi MA and Chand D. Complementary Feeding-Reasons for Inappropriateness in Timing, Quality and consistency, Indian $\mathrm{J}$ Pediatr 2008;75:49-56.

[18] Shumey A, Demissie M, Berhane Y.: Timely initiation of complementary feeding and associated factors among children aged 6 to 12 months in Northern Ethiopia: an institution-based cross-sectional study. BMC Public Health 2013 13:1050.

[19] Ethiopia Nutrition Profile of 2008. Nutrition and Consumer Protection Division, FARO, Nutrition Country Profile of the Federal Democratic Republic of Ethiopia. 2008.

[20] Kume A: Infant and Young Child Feeding Practices among Mothers Living In Harar, Ethiopia. Harar Bulletin of Health Sciences January 2012; Extracts Number 4, 66 -78.

[21] Senarath U, Sanjeeva S. P. Godakandage, Hiranya J,et al. Determinants of inappropriate complementary feeding practices in young children in Sri Lanka: secondary data analysis of Demographic and Health Survey 2006-2007. Maternal and Child Nutrition 2012; 8 (Suppl. 1): pp. 60-77.

[22] Upul S, Kingsley E. Agho, Dur-e-Samin A, et al. Comparisons of complementary feeding indicators and associated factors in children aged 6-23 months across five South Asian countries. Maternal and Child Nutrition 2012; 8 (Suppl. 1): pp. 89-106.

[23] Nira Joshi, Kingsley E. Agho, Michael J. Dibley, et al. Determinants of inappropriate complementary feeding practices in young children in Nepal: secondary data analysis of Demographic and Health Survey 2006. Maternal and Child Nutrition 2012; 8 (Suppl. 1): pp. 45-59.

[24] Disha A, Rawat R, Subandoro A, et al. Infant and Young Child Feeding (IYCF) Practices in Ethiopia and Zambia and their Association With Child Nutrition. African Scholarly Science Communication Trust April 2012; Volume 12 No.2: 5899-5900.

[25] Rose V, Surinder K Baines, Kingsley E Agho, Michael J Dibley. Factors associated with inappropriate complementary feeding practices among children aged 6-23 months in Tanzania. Matern Child Nutr. 2012 Aug 23; 22925557.

[26] Charmaine S Ng, Michael J Dibley, Kingsley E Agho. Complementary feeding indicators and determinants of poor feeding practices in Indonesia: a secondary analysis of 2007 Demographic and Health Survey data. Food Nutr Bull. 2010 Jun; 31 (2):366-75.

[27] Kimani-Murage et al.: Patterns and determinants of breastfeeding and complementary feeding practices in urban informal settlements, Nairobi Kenya. BMC Public Health 2011 11:396 